\title{
Diseño de un Jardín Botánico Universitario en áreas de piede- monte llanero: Campus Loma Linda, Universidad Santo Tomas, Villavicencio
}

\section{Design of a University Botanical Garden in Areas of the Los Llanos Foothills: Loma Linda Campus, Saint Thomas University, Villavicencio}

Rodrigo Isaac Velosa Caicedo ${ }^{1}$, Juan Manuel Gonzáles Trujillo ${ }^{2}$

\author{
1 Facultad de Ingeniería Ambiental, Universidad Santo Tomás, Villavicencio. \\ Email: rodrigovelosa@usantotomas.edu.co. \\ 2 Facultad de Ingeniería Ambiental, Universidad Santo Tomás, Villavicencio. \\ Email: juanmgonzalez@usantotomas.edu.co.
}

\section{OPEN ACCESS}

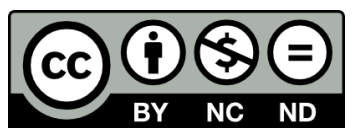

Copyright:(C) 2019 Ingenierías USBmed. La revista Ingenierías USBmed proporciona acceso abierto a todos sus contenidos bajo los términos de la licencia creative commons Atribución- no comercial- SinDerivar 4.0 Internacional (CC BY-NC-ND 4.0)

Tipo de Artículo: Investigación científica y tecnológica.

Recibido:13-06-2019.

Revisado: 01-08-2019.

Aprobado: 05-08-2019.

Doi: $\quad 10.21500 / 20275846.4152$

Referenciar así: R.I. Velosa \& J.M Gonzáles. "Diseño de un Jardín Botánico Universitario en áreas de piedemonte llanero: Campus Loma Linda, Universidad Santo Tomas, Villavicencio ". Ingenierías USBMed, 10(2), pp.31-43, 2019.

Declaración de disponibilidad de datos: Todos los datos relevantes están dentro del artículo, así como los archivos de soporte de información.

Conflicto de intereses: los autores han declarado que no existen conflicto de intereses.

Editores: Yohana López Rivera, Universidad de San Buenaventura, Medellín, Colombia. Alfonso Insuasti Rodríguez, Universidad de San Buenaventura, Medellín, Colombia. Erika Solange Imbett Vargas, Instituto Tecnológico Metropolitano. Eliana Zapata Ruiz, Instituto Tecnológico Metropolitano. José Fernando Valencia Grajales, Universidad Autónoma Latinoamericana.
Resumen. A través del tiempo, los jardines botánicos han evolucionado hasta convertirse en entornos de conservación de la biodiversidad; su evolución refleja no solo los cambios en los propósitos y deseos de conservación de culturas locales, sino también aquellos en los aportes conceptuales, metodológicos y técnicos de diversas disciplinas del conocimiento (incluidos los cambios de percepción en la manera de apreciar los entornos naturales). Los jardines botánicos universitarios suelen asociarse con fragmentos de vegetación natural de diversos tamaños, en diferentes etapas de sucesión ecológica y dentro del contexto de ecorregiones o zonas de vida específicas; también se vinculan con el mantenimiento de especies nativas (amenazadas, promisorias, ornamentales, etc.), y el desarrollo de actividades de educación, investigación y sensibilización ambiental. Este artículo es producto de una investigación realizada en la Universidad Santo Tomás, Sede Villavicencio, (Meta, Colombia), que tuvo por objeto evaluar la viabilidad ecológica y técnica del establecimiento de un área de jardín botánico en dicha institución: se pretende compartir las reflexiones realizadas en torno a la importancia, los retos y principales impactos de los jardines botánicos como estrategias para conservación de biodiversidad, así como ilustrar los propósitos de planificación y lineamientos de diseño de un jardín botánico universitario en la zona de piedemonte llanero.

Palabras Clave. Jardines botánicos, conservación de biodiversidad, lineamientos de diseño.

Abstract. Over time, botanical gardens have evolved up to a point in which they have become environments of biodiversity conservation. Their evolution does not only reflect changes in purposes and desires of conservation of local cultures, but also those in the conceptual, methodological and technical contributions from various disciplines of knowledge (including changes of perception in the way of appreciating the natural environment). University botanical gardens are often associated with fragments of natural vegetation of different sizes, in different stages of ecological succession and within the context of ecoregions or specific areas of life. They are also linked with the maintenance of native species (endangered, promising, ornamental, etc.), and the development of education, research, and environmental awareness activities. This article is a product research conducted at the University of Santo Tomas, Villavicencio campus, in Meta, Colombia. It was designed to evaluate the ecological and technical feasibility of the establishment of an area of a botanical garden in this institution. It aims to share the reflections made on the importance, challenges and major impacts from the botanical gardens as a strategy for conservation of biodiversity, as well as to illustrate the purposes of planning and designing guidelines for a University Botanical Garden in the area of the Los Llanos Foothills.

Keywords. Botanical gardens, biodiversity conservation, design guidelines. 


\section{Introducción}

Los jardines botánicos han sido definidos como instituciones que mantienen colecciones documentadas de plantas vivas con propósitos de investigación científica, conservación (in situ y ex situ), exhibición y educación $[1,2,3,4,5,6,7,8,9,10]$. Su historia es extensa, particularmente en países de Europa Occidental, Norteamérica y Asia; empero, en América Latina su historial y proceso de documentación son relativamente recientes, y gran parte de la misma puede ser monitoreada a partir de la creación, en 1808, del Jardín Botánico de Río de Janeiro (Brasil), destacado por su organización y calidad paisajística del entorno natural [11, 12]. En Colombia, por iniciativa del célebre naturalista Enrique Pérez Arbeláez, en 1955 se creó el Jardín Botánico de Bogotá "José Celestino Mutis", el cual ha sido uno de los más importantes en los procesos de conservación, investigación, integración e intercambio de información en el país [13]. Durante su corta evolución, los jardines botánicos en Latinoamérica y Colombia, particularmente los universitarios, han incorporado fragmentos de vegetación natural en diferentes etapas de sucesión ecológica, con actividades de identificación, catalogación y propagación de flora nativa amenazada, endémica y de algún interés en campos de la silvicultura, horticultura etc., incluidos también propósitos de ecoturismo guiado [13]. En la actualidad, algunos jardines botánicos han sido pensados como "centros de conservación" para la promoción, entre otros, de programas de investigación, educativos y de ecoturismo entre visitantes nacionales y extranjeros, tendientes a incrementar la sensibilidad sobre la conservación restauración de recursos ecosistémicos, florísticos y de fauna, y sobre los beneficios que ellos prestan a la gente en los ámbitos locales, regionales y nacionales.

Bajo esta perspectiva, los jardines botánicos en la actualidad, y en particular en regiones ricas en biodiversidad como el departamento del Meta, pueden jugar un papel clave en la expansión del mercado ecoturístico, lo cual puede estimular la generación de prácticas de conservación sostenible de recursos faunísticos y florísticos específicos de una región o territorio. Así, por ejemplo, si un jardín botánico en un área pequeña de la zona urbana de una ciudad ofrece la oportunidad a un visitante de conocer y apreciar la flora nativa específica y única de una región o territorio muy extenso, a la cual solo se podría acceder si se viaja a sitios muy remotos, se promocionaría la generación de actividades de conservación sostenibles.

\section{A. Diversidad de roles}

Los jardines botánicos son importantes por la función ecológica que pueden prestar: además de ser promotores de la educación ambiental, constituyen espacios para la conservación e investigación sobre la flora y fauna nativa, con lo que pueden integrarse al sistema ambien- tal estructurante de un municipio o de una localidad y contribuyen al ordenamiento y monitoreo ambiental de un territorio [14, 15, 16]. También pueden funcionar como proveedores de material vegetal para el desarrollo de actividades de restauración, reforestación, revegetalización y repoblamiento que necesiten los municipios $\mathrm{u}$ otras instancias regionales, y tienen impacto en la disminución de la huella de carbono generada por las áreas de poblamiento urbano.

Tal como se anotó, otro campo de participación de los jardines corresponde al desarrollo de actividades ecoturísticas mediante las cuales las personas pueden interactuar con el medio y, de esta manera, generar sentido de pertenencia por este capital natural. De acuerdo con Quintana [17], estas instituciones pueden ofrecer servicios tales como alquiler de salones, folletos realizados con resúmenes de investigaciones, venta de material vegetal, asesoría para el diseño de jardines, asistencia técnica en cultivos agroforestales, manejos de semillas, arborizaciones en predios privados y vegetación con fines ornamentales, establecimiento de cercas y servicio de guía por senderos interpretativos, entre otros.

De acuerdo con lo anterior, los jardines botánicos pueden jugar una diversidad de roles, los cuales pueden ser canalizados de manera efectiva hacia el fomento de la protección, uso y manejo sustentable de los recursos naturales en general.

\section{B. Diversidad de diseños}

Al igual que las funciones, el diseño de un jardín botánico también es muy variable, en tanto que refleja los cambios en la forma de percibir los entornos naturales que han tenido las culturas humanas locales a través del tiempo. Desde el siglo XIX los jardines botánicos fueron concebidos como áreas semejantes a parques, con énfasis en el desarrollo de actividades de recreación y esparcimiento [18]. Esta apreciación estética pervive en la actualidad, sumada al creciente interés de hacerlos más atractivos al público y de fortalecer, en el caso de los jardines botánicos universitarios, las actividades de conservación, investigación y educación. Hoy, los jardines botánicos tienden a tener una visión multifacética que aún gira en torno al papel ornamental de las plantas nativas, sumando su valor estético al de la educación e investigación y convirtiéndose en un motivo de orgullo para una comunidad local.

El diseño (tamaño, forma y aun la proximidad con otras áreas naturales vecinas) de un jardín debe ser coherente con sus propósitos misionales: en el caso particular de jardines botánicos universitarios, la versatilidad en cuanto a funcionalidad debe estar muy ligada a sus propósitos de investigación y educación, en el contexto de la programación e implementación de prácticas ecológicas sostenibles. 
C. Los jardines botánicos como centros de educación e investigación

Desde un jardín botánico se difunde respeto y amor hacia el medio ambiente y se participa de forma activa en actividades relacionadas con la conservación de biodiversidad, lo que incluye desde las preferencias de la gente para establecer la composición del jardín (por ejemplo en plantas medicinales, orquídeas, heliconias, palmas y plantas para horticultura) hasta procesos de restauración ecológica [5], [19, 20, 21, 22, 23]. En el contexto internacional, los jardines han jugado un papel importante en los esfuerzos de conservación de plantas; actualmente se debate su adhesión a los requerimientos del Protocolo de Nagoya sobre acceso a recursos genéticos [24]. Donaldson [25] propone que el eje central de los jardines botánicos sea la investigación en aspectos como planificación en conservación, modelamiento de la respuesta de especies al cambio climático, conservación de especies amenazadas y tests experimentales de cambio global.

Mediante el uso de la ciencia y la tecnología, los jardines botánicos pueden promover la conservación de especies amenazadas en los ámbitos local, regional y nacional. Algunos autores han documentado el papel de los jardines botánicos en la conservación de orquídeas amenazadas, en tanto que se involucran áreas de la ecología (como interacciones con polinizadores y asociaciones micorrizales) con tecnologías de genética de la conservación, propagación en bancos de germoplasma para reintroducciones y restauraciones, o reintroducciones de las especies amenazadas [3, 26].

En el largo plazo, las colecciones de plantas nativas ex situ que se pueden mantener en un jardín botánico proveen una oportunidad para incrementar la adaptabilidad de las plantas a cambios medioambientales, de manera tal que se incrementen las posibilidades de éxito en futuras reintroducciones de las especies a los hábitats naturales donde han sido disminuidas [27, 28]. Esta oportunidad, que implica un manejo de la deriva genética y de los procesos de selección ex situ, constituye una valiosa herramienta para prevenir la extinción de especies que se encuentran amenazadas [27, 29].

Según algunos autores, las acciones de conservación fuera del hábitat, o condiciones ex situ, deben realizarse para un conjunto de especies o taxas que cumplan con las siguientes condiciones [30]:

1. Especies pertenecientes a taxas que se encuentran en riesgo inmediato de extinción, ya sea a nivel local, nacional o global.

2. Especies pertenecientes a taxas que son de importancia económica local, como las plantas cultivadas comestibles, medicinales, y silvestres o cultivadas que proveen la base de la industria, agricultura, horticultura y artesanías locales.

3. Especies o subespecies "bandera" de una localidad que pueden estimular la conciencia sobre la conservación y ser incorporadas dentro de los programas de educación y gestión ambiental.

4. Especies o taxas que son de especial interés científico, tales como las asociadas a endemismos reducidos o relictos geográficos muy específicos [23].

Igualmente, la conservación dentro del hábitat - o condición in situ - forma parte del quehacer de algunos jardines botánicos. Sin embargo, aquellos que poseen colecciones in situ en áreas fragmentadas pueden presentar problemas en materia de viabilidad de las especies en el largo plazo de no establecerse conectividades regionales que permitan el intercambio de especies, a pesar de su importancia como refugios para la conservación de especies de flora y fauna, [31, 32]. En un estudio realizado en un área fragmentada de 1 ha de bosque húmedo tropical en el jardín botánico de Singapur se estableció que, si bien estas áreas actúan como refugios después del aislamiento, aún por décadas, no son garantías de viabilidad a largo plaz, debido a la ausencia de conectividades con el entorno regional que sean fuente de propágulos para el área aislada [31]. Asimismo, las áreas fragmentadas que forman parte de un jardín botánico se enfrentan al problema de la llegada y colonización por especies invasoras, generalmente exóticas, que pueden alterar la composición y diversidad de especies original presente en los fragmentos. En tales casos, los jardines botánicos requieren de un monitoreo permanente de sus colecciones vivas y ex situ para controlar la llegada de especies invasoras, tal como ha ocurrido en algunos de ellos en el ámbito mundial $[33,34]$.

El espectro de tipos de jardines botánicos es bastante amplio en la actualidad: existen desde grandes jardines que desarrollan un amplio rango de actividades, hasta instituciones pequeñas con recursos y actividades limitadas [35]. Sin embargo, sus ámbitos de acción son muy similares y todos juegan un papel importante en la conservación integral, el desarrollo sostenible y la educación.

\section{Los jardines botánicos y el ecoturismo}

Los jardines botánicos constituyen un referente para los municipios y sus habitantes; por consiguiente, su deber ser se asocia a un espacio abierto a visitantes y a todo tipo de actividades y demandas educativas, culturales y ecoturísticas $[18,36]$. Manteniendo bajo consideración principios educativos, de conservación y de investigación, algunos jardines botánicos hoy en día incorporan en sus diseños estéticos muestras de grupos funcionales de plantas, y aun de ecosistemas. Así, por ejemplo, el Jardín Botánico de Bogotá ha incorporado el ecoturismo a través del recorrido por espacios que recrean, en vivo, la amplia diversidad ecosistémica de Colombia. En este caso, la educación de los visitantes ha demostrado ser esencial para el 
éxito de los programas de conservación que lidera esta institución en particular.

\section{Elementos de diseño para jardines botáni- cos: el caso del campus Loma Linda de la Universidad Santo Tomás (Villavicencio)}

En la literatura se reporta una gran diversidad de lineamientos de diseño aplicables a diferentes tipos de jardines botánicos. Se incluyen aspectos relacionados con la estructura de los jardines botánicos, la organización de las colecciones, los tipos de infraestructura, los servicios ofrecidos a los visitantes, etc. Sin embargo, y con el objeto de maximizar los esfuerzos de conservación de los jardines, los lineamientos de diseño deben ser específicos frente a los objetivos particulares del jardín.

La Universidad Santo Tomás (USTA) - Villavicencio (Colombia) cuenta con un relicto boscoso en el Campus Loma Linda, que constituye un área potencialmente estratégica para realizar acciones de conservación de la biodiversidad. Para el establecimiento del jardín, pensado como un campo de relación interdisciplinar entre las ciencias naturales, la ingeniería, la arquitectura y el urbanismo, los lineamientos de diseño se dividieron en las siguientes categorías:

1. Misión del jardín y oferta ambiental del sitio (plantas y aves)

2. Incorporación de hábitats de piedemonte

3. Incorporación de un sendero ecológico interpretativo

4. Re-funcionalización de la infraestructura existente

5. Incorporación de prácticas sostenibles en las actividades diarias

6. Articulación de la investigación con la educación

7. Acciones, servicios y políticas del jardín

A. Misión del jardín y oferta ambiental del sitio

La misión propuesta para el Jardín Botánico USTA Villavicencio radica en "contribuir a la conservación de la diversidad biológica de los ecosistemas de piedemonte llanero a través de la investigación y manejo de áreas de conservación, para generar y difundir conocimiento y ofrecer escenarios para educación y recreación". Las siguientes propuestas de planificación constituyen una contribución a los esfuerzos de conservación regionales y locales en apoyo de la misión establecida para el jardín botánico del Campus Loma Linda. Se incluyen una variedad de propuestas de diseño orientadas, de un lado, a la conservación regional de plantas de piedemonte y sabana de la Orinoquía; y de otro, a la introducción de técnicas que integren el desarrollo y los hábitats naturales, de suerte tal que se ilustre la belleza de las plantas nativas y la necesidad de protegerlas, y se brinden a visitantes y estudiantes de la universidad diferentes oportunidades de establecer vínculos con las plantas.
Para guiar el proceso de planificación y toma de decisiones de diseño del jardín se realizó una caracterización preliminar de la flora y fauna - aves del sitio, enfatizada en la definición de especies singulares, representativas y carismáticas, clave para la restauración activa del mismo. Se hizo un registro preliminar de 80 especies vegetales en total, representadas en 35 familias; la determinación taxonómica fue realizada por el dendrólogo Francisco Castro. Las familias con mayor número de especies fueron Leguminosae, Melastomataceae y Arecaceae (figura 1).

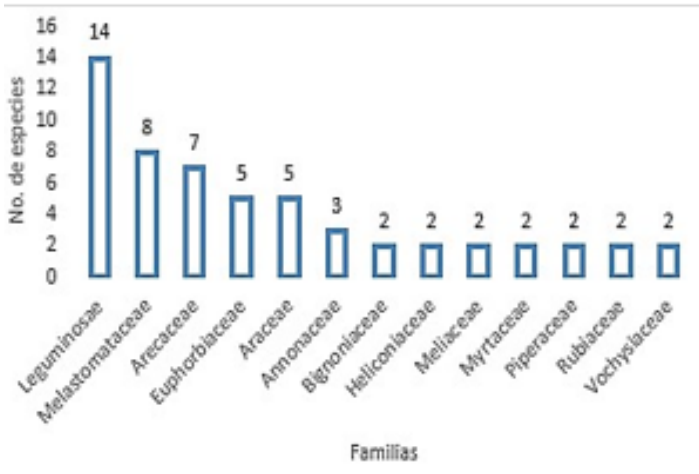

Figura 1. Familias de plantas con el mayor número de especies (riqueza) en el Campus Loma Linda

En su mayoría, las especies registradas son características de los ecosistemas del piedemonte llanero [37]. La ocurrencia de especies típicas de etapas tempranas de la regeneración natural pertenecientes a las familias Melastomataceae, Polygonaceae y Piperaceae evidencia estados iniciales de sucesión ecológica en algunos sitios. El registro de especies de rápido crecimiento como Cecropia engleriana (Yarumo), Scheflera marototoni (Pategallina), Miconia spp (tunos, varias especies), Vismia macrophylla (Siete cueros), Piper obliquum (Cordoncillo), Guarea guidonia (trompillo), Bellucia grossularioides (guayabo de pava) y Triplaris weigeltiana (Vara santa), así como la presencia de pastos exóticos en algunos sitios del interior del bosque, indican un grado de deterioro por actividades extractivas y de pastoreo realizadas en el pasado. Estas especies son plantas pioneras heliófilas y oportunistas que ocupan los claros abiertos por las actividades extractivas.

Por otra parte, también sobresale el registro de especies características de estados de sucesión intermedios, entre las que se destacan Alchornea triplinervia (carcomo, carne gallina), Alchorneopsis floribunda (carcomo, carne gallina), Cassia moschata (Cañafístola), Byrsonima spicata (Peralejo), Tapirira guianensis (Guarupayo), Enterolobium schomburgkii (orejero), Myrcia fallax (arrayán) y Clidemia hirta (mortiño). Estas especies se consideran pioneras intermedias en el avance sucesional. También se registran elementos pertenecientes a bosques maduros, entre los que se destacan Xylopia polyantha (escobillo), Annona pur- 
purea (tucuragua), Trattinnikia aspera (charaño), Marmaroxylon basijugum (rayadito), Swartzia trianae (frijolillo), y Andira surinamensis (almanegra). Tales especies conforman el dosel del bosque y sus frutos son consumidos por la fauna silvestre.

Se destaca la presencia de un número importante de especies de palma, entre las que se encuentran Euterpe precatoria (palma asaí), Socratea exorrhiza (palma choapo), Attalea butyracea (palma yagua), Attalea maripa (palma guichire), Oenocarpus bataua (palma seje), Oenocarpus minor (palma pusuy) y Geonoma interrupta (palmiche). Esta última es muy sensible a intervenciones antrópicas, por lo que se considera importante el establecimiento de colecciones vivas de esta especie y de otras palmas de sotobosque, como una colección viva del jardín botánico. Attalea maripa, por su parte, es una especie que se encuentra categorizada en bajo riesgo - LC en el libro rojo de palmas de Colombia. También es destacable la ocurrencia de Cedrela odorata (Cedro) en el área de estudio, la cual es una especie categorizada en peligro -EN de acuerdo con el libro rojo de plantas de Colombia. Por lo tanto, se considera importante prevenir la tala de esta especie en el área propuesta de jardín botánico, e incrementar la colección de la misma.

Respecto a la oferta de aves, se compiló información sobre la ocurrencia de 99 especies en el campus Loma Linda, las cuales corresponden a 35 familias. Este número, registrado de manera independiente por el biólogo Rodrigo Velosa y por la Corporación Autónoma Regional - Cormacarena, corresponde al 23,4 \% del total de especies de aves reportadas para la región de la Orinoquía [38]. Las familias con mayor riqueza de especies fueron Tyrannidae (Atrapamoscas, $\mathrm{n}=9$ ), Thraupidae (Azulejos y afines, $\mathrm{n}=8$ ), Picidae (Carpinteros, $n=6$ ), Turdidae (Mirlas, $n=6$ ), Emberizidae (semilleros, $n=6$ ), Columbidae (Torcazas, $n=4$ ) y Troglodytidae (Cucaracheros, $n=4$ ) (figura 2).

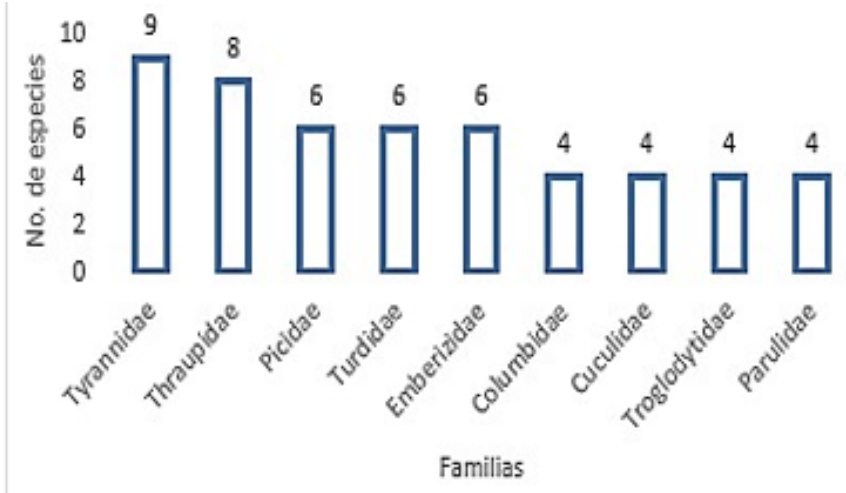

Figura 2. Familias de aves con el mayor número de especies (riqueza) en el campus Loma Linda

Se registraron 6 especies de aves migratorias latitudinales, referenciadas de la siguiente manera: Vireo olivaceus (verderón ojirojo), Catharus ustulatus (paraulata lomiaceituna), Piranga rubra (piranga roja), Dendroica striata (reinita rayada), Setophaga ruticilla (candelita norteña) y Setophaga petechia (reinita dorada). Estas especies realizan una migración latitudinal durante el periodo abril - diciembre hacia zonas bajas, en busca de alimentación. La mayor parte de las especies de aves registradas es de hábitos generalistas, no solo en cuanto al tipo de alimentación sino también con respecto al hábitat ocupado. Se destaca el bajo número de especies que son estrechamente dependientes del interior del bosque, lo cual constituye evidencia del grado de deterioro de la calidad del hábitat ocupado como consecuencia de la fragmentación.

No obstante, la alta riqueza de especies de aves reportada, al igual que la ocurrencia de algunas especies migratorias en el área de estudio y su zona de influencia, resaltan la importancia de la conservación de fragmentos de bosque secundario en diferentes etapas de sucesión ecológica, relativamente cercanos a otros de mayor tamaño dentro del cinturón verde del municipio de Villavicencio. Asimismo, es destacable la presencia de una alta riqueza de especies de la familia Thraupidae: esta se encuentra conformada por especies de hábitos esencialmente frugívoros con mezclas de insectos pequeños en la dieta; y que pueden jugar un papel esencial en la recuperación de áreas degradadas, dado su papel como dispersores de semillas. Entre estas especies se encuentran las siguientes:

Ramphocelus carbo (comequeso, cardenal), Thraupis episcopus (azulejo común), Thraupis palmarum (azulejo palmero) y Tangara cayana (tángara anteada). Otro grupo de especies dispersoras potenciales de semillas, en razón de que incorporan cantidades variables de frutos, son las siguientes: Euphonia laniirostris (eufonía gorgiamarilla, Fringillidae), Ortalis guttata (guacharaca, Cracidae), Columba cayannensis (torcaza morada, Columbidae), Cyanocorax violaceus (chare, Corvidae), Catharus nudigenis (mirla de ojos amarillos, Turdidae), Turdus ignobilis (mirla embarradora, Turdidae), Turdus albicollis (mirlo coliblanco, Turdidae), Turdus leucomelas (mirla buchiblanca, Turdidae), Mimus gilvus (mirla común, Mimidae), Cyanerpes cyaneus (mielero patirojo, Thraupidae) y Cyanerpes caeruleus (mielero púrpura, Thraupidae).

\section{B. Incorporación de hábitats de piedemonte}

El diseño del jardín engloba la incorporación de dos fragmentos boscosos de vegetación nativa, en un estado diferencial de avance de la sucesión ecológica y aislados uno de otro por áreas de pastizal, que proveen el hábitat para diferentes especies de flora y fauna. Tales fragmentos constituyen el área núcleo del jardín y promueven la conectividad con las áreas boscosas vecinas al campus. También se ha considerado la inclusión de senderos arbolados ya construidos que, además de brindar som- 
bra a los potenciales visitantes, constituirían lugares de recorrido donde se aprecie el inventario florístico y faunístico del jardín, y se puedan desarrollar actividades de educación e interpretación ambiental a cargo de estudiantes capacitados con anterioridad.

El aislamiento de los fragmentos boscosos por áreas de pastizal arbolado constituye un escenario para la implementación de estrategias de restauración que busquen superar las barreras a la regeneración natural después de un disturbio en áreas de piedemonte. Cuando el disturbio es muy intenso se pueden perder los mecanismos que tiene el sistema para regenerarse (lluvia de semillas, banco de semillas, bancos de plántulas, etc.), hecho que demandaría un mayor esfuerzo en los planes de restauración. En la restauración ecológica se trata de obtener toda la información posible respecto al estado pre-disturbio, con el fin de recuperar la mayor cantidad de elementos estructurales del ecosistema original $[39,40,41]$.

Los espacios propuestos para la realización de actividades de restauración ecológica incluyen tres áreas, a saber:

1. Zona de restauración con enriquecimiento con un área de 7.73 ha (fragmentos de bosque secundario en etapa temprana de sucesión ecológica).

2. Zona de restauración activa con un área de 2.15 ha (sitios para nucleación + instalación de perchas artificiales para aves).

3. Zona de rehabilitación ecológica con área de 2.41 ha (recuperación de suelo + reforestación con especies nativas).

La elaboración del mapa temático se realizó con información primaria del levantamiento topográfico y estos datos se vectorizaron mediante ArcMap 10.2.2. La conservación de estas áreas y el establecimiento de conectividades entre ellas a través de procesos de restauración y rehabilitación constituyen la guía para las decisiones de planificación y manejo del jardín en el largo plazo.

Con el objeto de definir el potencial del banco de semillas en acciones de restauración ecológica (natural o asistida) del sitio, se realizó un estudio comparativo para determinar la composición, distribución vertical, densidad y diversidad del banco de semillas germinable en áreas de pastizal y bosque secundario fragmentado del predio Loma Linda. Aunque diversos estudios han evaluado el efecto del tipo de vegetación sobre el banco de semillas en suelo, principalmente en ecosistemas andinos y páramos [42, 40, 41, 43], ninguno ha evaluado específicamente su influencia en áreas urbanas del piedemonte llanero [43]. Velosa (2018) reporta un cambio, aunque no significativo en términos estadísticos, en la composición y diversidad del banco de semillas en fragmentos de pequeña extensión y los pastizales adyacentes al campus Loma Linda [44]. Los cambios en la cobertura conducen a una mayor densidad y diversidad de semillas en áreas de pastizal, y a un cambio en la composición del banco de semillas, que incluyen la presencia de plantas herbáceas y otras especies ruderales, no solo al interior del pastizal sino también al interior de los pequeños fragmentos de bosque secundario. Tal presencia de plantas herbáceas de sucesión temprana es de particular interés, ya que fácilmente podrían extenderse con el incremento de la presión urbanística y ocasionar un retroceso en los procesos sucesionales. Por consiguiente, el enriquecimiento de los pequeños fragmentos de bosque secundario con especies de edades intermedias o avanzadas en el proceso de sucesión, sería clave para impedir la degradación completa de tales áreas. Adicionalmente, la conectividad de los pequeños fragmentos con fuentes semilleras provenientes de bosques vecinos también sería una contribución para aumentar la resiliencia de las áreas de piedemonte en conjunto.

\section{Incorporación de un sendero ecológico interpretativo}

El diseño del jardín incluye un espacio natural a manera de sendero ecológico interpretativo como oportunidad para recreación, educación ambiental y ecoturismo [45]. Para su construcción se requiere realizar una serie de valoraciones previas por las zonas donde se realizará el trazado, con el objeto de prevenir y evitar alteraciones al ecosistema tales como erosión y compactación del suelo, interferencia con las actividades reproductivas de aves y mamíferos, alteración de vegetación representativa, acumulación de basuras, contaminación de fuentes hídricas, y otros factores [46]. La falta de planificación del sendero puede traer como consecuencia una alteración del medio físico y una disminución en la calidad de experiencia de los futuros visitantes [46].

Para establecer el sendero ecológico interpretativo se realizó un análisis de la heterogeneidad del entorno y se buscaron alternativas para compatibilizar el uso del área con la conservación de biodiversidad; una herramienta para el manejo de la actividad ecoturística en un sendero público es el establecimiento de la capacidad de carga [47]. El estimativo de la capacidad de carga permite hacer una recreación controlada con un mínimo impacto ambiental. Sin embargo, estimar la capacidad de carga de un sendero exige saber de qué manera varían las características físicas como la erodabilidad, el grado de compactación del suelo, la pendiente y el anegamiento del terreno, a fin de generar factores de corrección con respecto al límite máximo de visitantes que pueden circular a través del sendero [47]. Dada la heterogeneidad del terreno, las anteriores características pueden variar de un sitio a otro a lo largo del sendero, generando así diferencias en las acciones de planificación y manejo que puedan establecerse a lo largo del mismo. 
El predio actual del campus Loma Linda de la Universidad Santo Tomás - Villavicencio donde se ubica el proyecto de jardín botánico tiene un área total de 16,48 ha. El área del jardín incluida en la propuesta es de 12,29 ha (75\% del área total del campus) y las coordenadas elipsoidales del jardín proyectado se ubican en el siguiente punto central: latitud N $4^{\circ} 06^{\circ} 38.50^{\prime \prime}$; longitud W $73^{\circ} 39^{\prime} 28.96^{\prime \prime}$. El trazado del sendero tiene una longitud de $1262 \mathrm{~m}$ en un área de 12,3 ha (figura 3); y la capacidad de carga real, estimada de acuerdo con el espacio disponible y luego de la aplicación de factores de corrección (social, accesibilidad, erodabilidad, anegamiento, compactación del suelo, impacto sobre fauna e impacto sobre flora) fue de 167 visitas por día, que grupos de 11 personas - incluido el guía - podrían realizar entre 8:00 a. m. y 4:00 p. m.

La determinación de la capacidad de carga real generada en este estudio es el primer paso para proyectar, a mediano y largo plazos, la creación de un jardín botánico en el campus Loma Linda que tenga asociado un sendero ecoturístico. Un uso regular del mismo con un horario establecido, un determinado número de personas por grupo, el acompañamiento de un guía para realizar la interpretación del sendero, la construcción de adecuaciones no invasivas para el descanso y discusión de temas ambientales durante el recorrido, la articulación de la actividad ecoturística al diseño curricular de los programas académicos de ingeniería ambiental y administración agropecuaria, constituyen elementos que aportan a la sostenibilidad ambiental del campus Loma Linda.

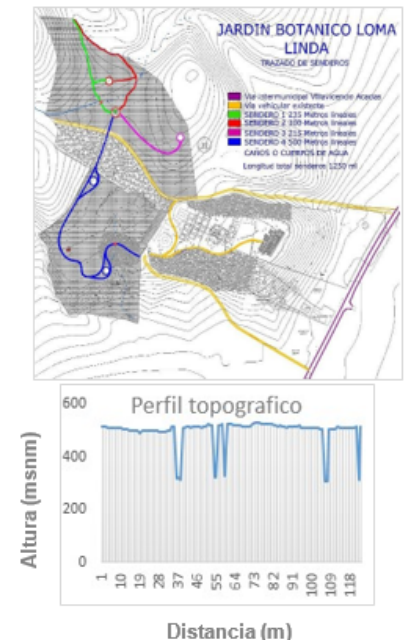

Figura 3. Trazado y perfil topográfico del sendero ecológico interpretativo propuesto para el Jardín Botánico USTA Villavicencio

En la figura 4 se ilustra una de las intervenciones proyectadas para el jardín relacionada con las estancias para interpretación ambiental a lo largo del sendero. Esto es, pequeños recintos construidos mediante arquitectura no invasiva y con la utilización de biomateriales (roca, madera rolliza, biomasa, cubierta verde) que faciliten la actividad de interpretación con el menor impacto posible en el entorno, dada su fragilidad.

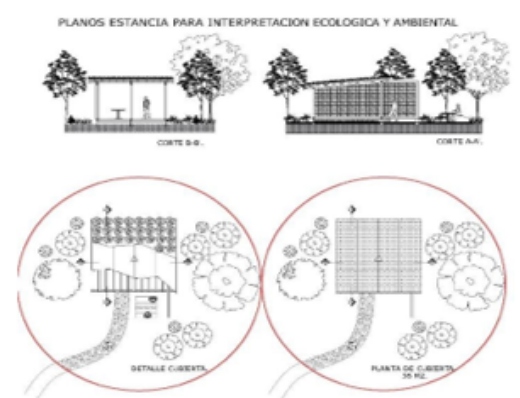

Figura 4. Esquema de intervenciones proyectadas para el sendero ecológico interpretativo del Jardín Botánico USTA Villavicencio

\section{Refuncionalización existente de la infraestructura}

La refuncionalización de espacios ha sido definida como el proceso de recuperación de una edificación existente, en el cual se transforman y modifican sus espacios para ajustarla a las nuevas necesidades [48]. La refuncionalización permite revitalizar el edificio mediante la alteración de su estado original y el cambio del uso para el que fuera diseñado; al refuncionalizar se recupera, se rescata y, sobre todo, se evita generar nuevos desechos de construcción con su posible demolición [48].

El diseño del jardín incluye la recuperación, reutilización y refuncionalización de parte de la infraestructura existente en el campus Loma Linda: así entonces, se revaloriza rehabilita y refuncionaliza la edificación, ajustándola a las necesidades del proyecto del jardín botánico. El diseño de los nuevos espacios parte de las medidas de los elementos, aulas de clase y espacios recuperados. Se propone convertir las edificaciones existentes relacionadas con aulas de clase, de carácter prefabricado, en áreas de vivero, semillero, bodegaje y espacios administrativos; también se proyecta un espacio dedicado al estudio, conservación e investigación. La propuesta de refuncionalización de la infraestructura existente para el proyecto de jardín botánico del campus se sintetiza a continuación (figura 5):

1. Zona de acceso vehicular y recepción $\left(600 \mathrm{~m}^{2}\right)$.

2. Zona de parqueo con capacidad para 28 vehículos $\left(900 \mathrm{~m}^{2}\right)$.

3. Zona de invernadero, no construida en la actualidad $\left(120 \mathrm{~m}^{2}\right)$

4. Zona de vivero, semillero y bodegaje (primera línea de aulas de clase en la actualidad, para refuncionalizar) $\left(450 \mathrm{~m}^{2}\right)$.

5. Zona para investigaciones y mantenimiento de colecciones biológicas ex situ (segunda línea de aulas en la actualidad, para refuncionalizar) $\left(450 \mathrm{~m}^{2}\right)$.

6. Zona para educación ambiental (tercera línea de 


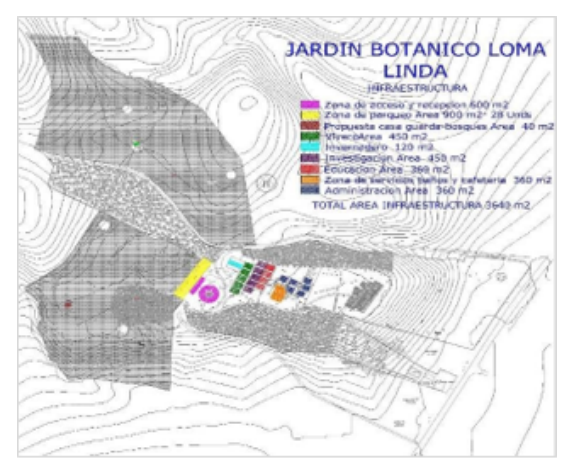

Figura 5. Propuesta de refuncionalización de parte de la infraestructura existente del campus Loma Linda

aulas de clase en la actualidad, para mantener) $\left(360 \mathrm{~m}^{2}\right)$.

7. Zona de servicios: tienda de recuerdos, librería, cafetería y baños (para adecuar) $\left(360 \mathrm{~m}^{2}\right)$.

8. Zona administrativa (cuarta, quinta y sexta línea de aulas de clase en la actualidad, para mantener) $\left(360 \mathrm{~m}^{2}\right)$.

E. Incorporación de prácticas sostenibles en las actividades diarias

La propuesta de creación de un área de jardín en el campus Loma Linda intenta demostrar un compromiso por la conservación de biodiversidad en áreas de piedemonte llanero, la aplicación de principios de diseño sostenible en todos los servicios e infraestructura, y la implementación de prácticas operativas sostenibles. En la figura 6 se ilustran algunas imágenes de intervenciones sostenibles para su implementación en el proyecto de jardín botánico.

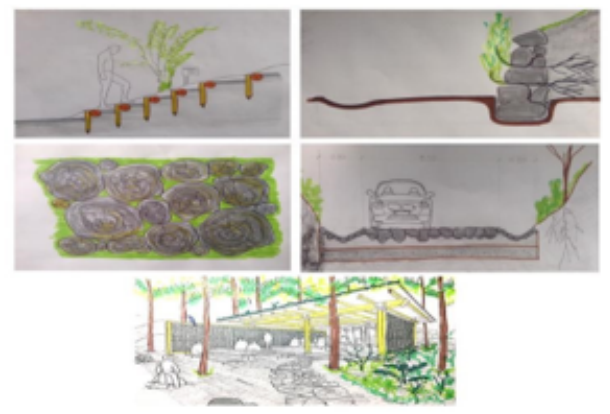

Figura 6. Imágenes ilustrativas para la ejecución de intervenciones sostenibles en el Jardín Botánico USTA Villavicencio

El uso de materiales como roca y madera facilitan realizar intervenciones de tipo no invasivo, en tanto que se evita al máximo la utilización de concreto y materiales que contravengan la naturaleza del proyecto (figura 6). La roca (piedra) como elemento de diseño y material de construcción tiene grandes ventajas y características propias; puede tener un impacto significativo en la calidad de vida de las personas. Esta puede in- tegrarse a cualquier tipología arquitectónica urbana: desde una casa de habitación hasta un complejo urbano. Su estética es capaz de causar fenómenos hermenéuticos relacionados con la identidad social, así como connotar un valor superior de calidad constructiva relacionado con la arquitectura vernácula [49].

Para la implementación del vivero no se utilizarán pesticidas ni fertilizantes inorgánicos químicos. La cuenca que suministra agua para el saneamiento básico del campus Loma Linda genera caudales en los diferentes periodos de retorno (5, 10, 25 y 50 años) del orden de 25 a $90 \mathrm{l} / \mathrm{s}$, suficientes para satisfacer las necesidades actuales del campus y los proyectados para el área del jardín [45].

\section{F. Articulación entre investigación y educación}

La creación del Jardín Botánico de la Universidad Santo Tomás de Villavicencio ofrecerá a estudiantes, docentes y al resto de visitantes la oportunidad de estudiarlo desde diferentes puntos de vista: adaptaciones de las plantas al medio, flora endémica, especies amenazadas, etnobotánica, horticultura etc. Ello permitirá, entre otros aspectos, el fortalecimiento de la estructura ecológica de la ciudad de Villavicencio; además, el uso del jardín por parte de la comunidad universitaria puede tener distintos fines - didáctico, de investigación y recreativo-.

Senderos ecológicos como el propuesto constituyen una oportunidad de educar mediante la interpretación ambiental, entendida esta última como herramienta que establece una relación de comunicación entre el jardín y el visitante [50]. En un jardín botánico, la interpretación está diseñada para mejorar la calidad de la experiencia recreativa del visitante, así como para inspirar de una forma agradable, a través de sus colecciones, un mayor conocimiento y aprecio por la naturaleza.

El sendero propuesto es de doble vía, rodeado por árboles que brindarán sombra y frescura a los visitantes del jardín, y a lo largo del recorrido el visitante encontrará lugares de estancia para descansar y admirar la belleza de este espacio natural. En el recorrido por el jardín, los visitantes podrán apreciar todo su inventario botánico.

Los senderos pueden mejorar de forma potencial los objetivos de conservación y aumentar las oportunidades sociales [51], con un mínimo de impactos sobre el ambiente biofísico que atraviesa el sendero.

\section{G. Acciones, servicios y políticas}

Las acciones para desarrollar en el jardín botánico USTA Villavicencio son las siguientes:

1. Realizar investigación científica en conservación y restauración de hábitats degradados en zonas de piedemonte y humedales.

2. Colaborar con otros jardines botánicos de los ámbitos regional y nacional para efectos de intercam- 
bio de información científica.

3. Disminuir la pérdida de especies de plantas y de su diversidad genética mediante el mantenimiento de colecciones in situ y ex situ.

4. Producir material vegetal para la propagación de especies amenazadas de extinción de la Orinoquía.

5. Elaborar programas de educación ambiental.

6. Realizar capacitaciones en áreas afines a la ecología y la botánica.

7. Realizar entrenamiento a docentes y estudiantes en áreas de ecología y botánica.

8. Participar en reintroducciones de plantas a su medio natural.

9. Realizar investigación, conservación y manejo in situ y ex situ de colecciones de plantas silvestres.

Los servicios para ofrecer son:

1. Venta de plantas forestales y ornamentales

2. Venta de tierra abonada

3. Venta de suvenires

4. Asistencia técnica en proyectos de restauración ecológica, cultivos agroforestales, arborización en predios privados y públicos, y establecimiento de cercas vivas

5. Capacitaciones en permacultura

Las políticas del jardín se resumen de la siguiente manera:

1. Está integrado por todas las colecciones in situ y ex situ que lo conforman dentro del campus Loma Linda.

2. Trabaja en cooperación con la Red Nacional de Jardines Botánicos y otros jardines botánicos del municipio de Villavicencio y de la región.

3. Promueve códigos de conducta relacionados con la conservación y el uso sostenible de recursos silvestres, y en particular de plantas nativas, en su medio natural.

4. Está conformado por un director adscrito a la Facultad de Ingeniería Ambiental, responsable de orientar las actividades del jardín en conjunto con el decano de la facultad y el comité de apoyo científico.

5. Está conformado por un comité de apoyo integrado por un ingeniero forestal y un educador ambiental, encargados de brindar orientación técnica para el mantenimiento y desarrollo del jardín, lo mismo que para el manejo de las actividades educativas y de extensión que se programen.

6. Cuenta con un viverista, encargado de la propagación y manejo de plántulas en el vivero.

7. Facilita el acceso a universidades y centros de investigación científica interesados en los campos de la conservación y restauración ecológica.

8. Desarrolla planes y programas de conservación, in- vestigación y educación ambiental a escalas local y regional en conjunto con la facultad de Ingeniería Ambiental.

9. Ejerce sus actividades con el apoyo de los programas de Ingeniería Ambiental y de Administración de Empresas Agropecuarias en temas de investigación, producción sostenible y educación e interpretación ambiental.

\section{Conclusiones}

El plan conceptual bajo el cual se propone la distribución de los componentes paisajísticos y de infraestructura del jardín botánico USTA Villavicencio incluye los siguientes ítems:

1. Zona de acceso y recepción

2. Zona de parqueo

3. Casa de guarda parque

4. Zona de invernadero

5. Zona de vivero - germinación

6. Zona de investigación - educación

7. Zona administrativa

8. Zona de servicios, baños y cafetería

9. Zona de sendero ecológico y estancias para interpretación ambiental

Esta propuesta enfatiza la conservación de la biodiversidad y la interpretación de los sistemas ecológicos locales como columna vertebral en las decisiones de planificación del campus Loma Linda, y crea un fundamento sólido bajo el cual se difunde un mensaje de prácticas de sostenibilidad.

Las fortalezas de esta propuesta se fundamentan en los siguientes ítems:

1. Propiedad del predio propuesto como Jardín Botánico: el área de estudio forma parte de un predio propiedad de la Universidad Santo Tomás, ubicado en la siguiente dirección: carrera 48 n. ${ }^{\circ}$ 19-45 Sur, barrio Loma Linda, Comuna 8, vereda Montecarlo, en jurisdicción del municipio de Villavicencio, departamento del Meta. Se localiza sobre las estribaciones del piedemonte de la cordillera oriental, sobre la margen derecha de la vía que conduce de Villavicencio a Acacías; limita con el barrio Villa Lorena al occidente, y al sur con el barrio Playa Rica. El área de estudio forma parte del cinturón verde del piedemonte, donde se establecen usos del suelo dedicados a la protección en la totalidad del espacio; forma parte de las cuencas de los ríos Ocoa y Guatiquía, y se localiza en el ecosistema de bosque húmedo subandino.

2. Acceso: al área de estudio dispone de una sola vía de acceso peatonal y dos vehiculares, que rodean los flancos norte y parte del sur del predio. Estas rutas permiten un buen control y están habilitadas 
durante todo el año.

3. Infraestructura: se cuenta con alguna infraestructura ya construida que podría sustentar algunas actividades que se demandarían, tales como cafetería y sitio para venta de suvenires. Existe también otra infraestructura construida en el sistema Royal Andina, representada por 19 salones de clase que comprenden un área aproximada de $7353,4 \mathrm{~m}^{2}$, que puede ser refuncionalizada para los propósitos de un Jardín Botánico con los siguientes fines: sitio para vivero bajo el sistema de invernadero; sitio para investigación e instalación de colecciones biológicas ex situ; sitio para educación ambiental; y sitio para oficinas administrativas del jardín.

4. 4. Ubicación dentro del cinturón verde de Villavicencio. Además de ser un área forestal protectora, el lugar en el que se localiza el área de estudio conforma un corredor biológico de vital importancia para la ciudad. Según el Plan de Ordenamiento Territorial de Villavicencio del año 2015, en este corredor se establecen franjas de suelo dispuestas transversalmente al área urbana municipal, que tienen como propósito principal "restituir los flujos naturales de materia y energía; así como, brindar condiciones benéficas para la sostenibilidad de la capacidad de auto regulación hídrica; la preservación y el repoblamiento de la vida silvestre, vegetal y/o animal" [52].

De la misma manera, para estas áreas están asignados usos forestales que cumplen la función de contener la presión de la expansión urbana sobre el área del piedemonte del Municipio de Villavicencio.

5. Único Jardín Botánico Universitario en Villavicencio: el área de estudio constituye el único jardín botánico que sería manejado por una entidad universitaria en el municipio.

6. Oferta ambiental. Para el área de estudio se identifican cinco tipos de coberturas vegetales que soportan la biodiversidad y servicios ambientales generados por el área: cerca de 100 especies de aves registradas y un pool de especies de plantas que, además de los beneficios ecológicos y ambientales, podrían ser utilizadas para efectos de rehabilitación de áreas abiertas de piedemonte, enriquecimiento de áreas de bosque secundario, palmetum y jardinería.

Esta propuesta de creación de un jardín botánico universitario constituye un proyecto piloto que tiene como metas acercar a los jóvenes universitarios, y en general, a los habitantes de Villavicencio, al uso sostenible de las áreas naturales de piedemonte fuertemente amenazadas por la creciente urbanización, y generar a su vez espacios recreativos y de investigación donde la comunidad genere conocimiento y dialogue, con lo que se disminuirían las actitudes de violencia y aquellas no compatibles con la conservación. Igualmente, la iniciativa correspondería a una estrategia de afianzamiento de la estructura ecológica principal del municipio que empezaría a integrar dos jardines botánicos de la ciudad con el resto de las áreas protegidas del municipio y de la región.

Bajo este contexto, es esencial recordar la conexión entre la misión del jardín y los lineamientos de diseño: lo misional de un jardín botánico debe ser la guía que oriente todas las decisiones de planificación y diseño, y las decisiones de diseño deben apoyar lo misional.

\section{Referencias}

[1] P. W. Jackson and L. A. Sutherland, "Role of botanic gardens," Encyclopedia of Biodiversity, vol. 6, pp. 504-521, 2013.

[2] Botanic Gardens Conservation International, "Botanic garden." [On line]. Available: htpps://www.bcgi.org, 2012.

[3] N. D. Swarts and K. W. Dixon, "Perspectives on orchid conservation in botanic gardens," Trends Plant Sci, vol. 14, no. 11, pp. 590-598, 2009.

[4] S. F. Oldfield, "Botanic gardens and the conservation of tree species," Trends in plant science, vol. 14, no. 11, pp. 581-583, 2009.

[5] J. Chen, C. H. Cannon, and H. Hu, "Tropical botanical gardens: at the in situ ecosystem management frontier," Trends in Plant Science, vol. 14, pp. 584-589, 2009.

[6] L.-S. Chang, R. J. Bisgrove, and M.-Y. Liao, "Improving educational functions in botanic gardens by employing landscape narratives," Landscape and Urban Planning, vol. 86, no. 3-4, pp. 233-247, 2008.

[7] M. Soderstrom, "Botanical gardens," 2008.

[8] A. Olaya, A. Rivera, and C. Rodríguez, "Plan nacional de colecciones para los jardines botánicos de colombia," Red Nacional de Jardines Botánicos de Colombia y Ministerio del Medio Ambiente. Bogotá, Colombia, pp. 1-41, 2002.

[9] Instituto Alexander Von Humboldt-IaVH Plan $\mathrm{Na}$ cional de jardines botánicos de Colombia, Red $\mathrm{Na}$ cional de Jardines Botánicos, Darwin Initiative, 2001.

[10] P. Wyse Jackson and L. Sutherland, "Agenda internacional para la conservación en jardines botánicos," Organización Internacional para la Conservación en Jardines Botánicos (BGCI), UK, 2000.

[11] Botanic Gardens Conservation International, "6th global botanic garden congress," 2017.

[12] R. R. Rodríguez, Martín Cárdenas, el eximio botánico y naturalista de América. La Paz: Plural 
editores, 2005.

[13] M. Pabón, "Jardines botánicos universitarios en la universidad estatal del centro-occidente," Ciencias Humanas, vol. 33, pp. 99-118, 2004.

[14] R. Mathieu, C. Freeman, and J. Aryal, "Mapping private gardens in urban areas using objectoriented techniques and very high-resolution satellite imagery," Landscape and Urban Planning, vol. 81, no. 3, pp. 179-192, 2007.

[15] M. Maunder, S. Higgens, and A. Culham, "The effectiveness of botanic garden collections in supporting plant conservation: a european case study," Biodiversity \& Conservation, vol. 10, no. 3, pp. 383401, 2001.

[16] M. Maunder, B. Lyte, J. Dransfield, and W. Baker, "The conservation value of botanic garden palm collections," Biological Conservation, vol. 98, no. 3, pp. 259-271, 2001.

[17] R. Quintana, "Bases para la implementación de un sistema de gestión ambiental para el jardín botánico universidad tecnológica de pereira, pereira." Tesis de pregrado, UTP, Pereira, 2005.

[18] P. A. Kumble and C. C. Houston, "The elements of a conservation botanic garden for ecotourism: Belize botanic garden as a case study," Journal of Landscape Studies, vol. 2, pp. 1-15, May 2009.

[19] L. M. Pearce, A. Davison, and J. B. Kirkpatrick, "Personal encounters with trees: The lived significance of the private urban forest," Urban Forestry \& Urban Greening, vol. 14, no. 1, pp. 1-7, 2015.

[20] D. Kendal, K. J. Williams, and N. S. Williams, "Plant traits link people's plant preferences to the composition of their gardens," Landscape and Urban Planning, vol. 105, no. 1-2, pp. 34-42, 2012.

[21] K. A. Hardwick, P. Fiedler, L. C. Lee, B. Pavlik, R. J. Hobbs, J. Aronson, M. Bidartondo, E. Black, D. Coates, M. I. Daws, et al., "The role of botanic gardens in the science and practice of ecological restoration," Conservation Biology, vol. 25, no. 2, pp. 265-275, 2011.

[22] C. D. Ward, C. M. Parker, and C. M. Shackleton, "The use and appreciation of botanical gardens as urban green spaces in south africa," Urban Forestry $\&$ Urban Greening, vol. 9, no. 1, pp. 49-55, 2010.

[23] Organización Internacional para la conservación en Jardines Botánicos, "Agenda internacional para la conservación en jardines botánicos," 2001.

[24] K. Davis, M. F. Smit, M. Kidd, S. Sharrock, and P. Allenstein, "An access and benefit-sharing awareness survey for botanic gardens: Are they prepared for the nagoya protocol?," South African Journal of Botany, vol. 98, pp. 148-156, 2015.

[25] J. S. Donaldson, "Botanic gardens science for conservation and global change," Trends in plant sci- ence, vol. 14, no. 11, pp. 608-613, 2009.

[26] M. M. Ramsay and K. W. Dixon, "Propagation science, recovery and translocation of terrestrial orchids," Orchid conservation, pp. 259-288, 2003.

[27] A. Ensslin, O. Tschöpe, M. Burkart, and J. Joshi, "Fitness decline and adaptation to novel environments in ex situ plant collections: current knowledge and future perspectives," Biological conservation, vol. 192, pp. 394-401, 2015.

[28] M. Maunder, "Plant reintroduction: an overview," Biodivers. Conserv, vol. 1, no. 1, pp. 51-61, 1992.

[29] A. C. Basey, J. B. Fant, and A. T. Kramer, "Producing native plant materials for restoration: 10 rules to collect and maintain genetic diversity," Native Plants Journal, vol. 16, no. 1, pp. 37-53, 2015.

[30] M. Lascurian, O. Gómez, O. Sánchez, and C. Hernández, Jardines Botánicos: Conceptos, operación y manejo. México: Asociación Mexicana de Jardines Botánicos, 2006.

[31] I. Turner, K. Chua, J. Ong, B. Soong, and H. Tan, "A century of plant species loss from an isolated fragment of lowland tropical rain forest," Conservation biology, vol. 10, no. 4, pp. 1229-1244, 1996.

[32] Y. van Heezik and K. Ludwig, "Proximity to source populations and untidy gardens predict occurrence of a small lizard in an urban area," Landscape and urban planning, vol. 104, no. 2, pp. 253-259, 2012.

[33] S. L. Sharrock, "The biodiversity benefits of botanic gardens," Trends in ecology $\&$ evolution, vol. 26, no. 9, p. 433, 2011.

[34] P. E. Hulme, "Addressing the threat to biodiversity from botanic gardens," Trends in Ecology \&6 Evolution, vol. 26, no. 4, pp. 168-174, 2011.

[35] A. Vovides and C. Hernández, en Jardines Botánicos, conceptos, operación y manejo. México: Asociación Mexicana de Jardines Botánicos, 2006.

[36] C. L. Wassenberg, M. A. Goldenberg, and K. E. Soule, "Benefits of botanical garden visitation: A means-end study," Urban Forestry \& Urban Greening, vol. 14, no. 1, pp. 148-155, 2015.

[37] J. E. Carvajal-Cogollo and J. N. Urbina-Cardona, "Patrones de diversidad y composición de reptiles en fragmentos de bosque seco tropical en córdoba, colombia," Tropical Conservation Science, vol. 1, no. 4, pp. 397-416, 2008.

[38] O. Acevedo-Charry, A. Pinto-Gómez, and J. O. Rangel-Ch, "Las aves de la orinoquia colombiana: una revisión de sus registros," Colombia Diversidad Biótica XIV. La región de la Orinoquía de Colombia, vol. 14, pp. 691-750, 2014.

[39] A. Díaz, Variación espaciotemporal de la lluvia de semillas en pastizales de un ecosistema alto andino 
(Reserva Forestal Municipal de Cogua, Cundinamarca). 2004.

[40] E. E. Santo-Silva, W. R. Almeida, M. Tabarelli, and C. A. Peres, "Habitat fragmentation and the future structure of tree assemblages in a fragmented atlantic forest landscape," Plant ecology, vol. 217, no. 9, pp. 1129-1140, 2016.

[41] K. Valenta, T. S. Steffens, R. R. Rafaliarison, C. A. Chapman, and S. M. Lehman, "Seed banks in savanna, forest fragments, and continuous forest edges differ in a tropical dry forest in madagascar," Biotropica, vol. 47, no. 4, pp. 435-440, 2015.

[42] T. R. Sousa, F. Costa, T. Bentos, N. L. Filho, R. C. G. Mesquita, , and I. O. Ribeiro, "The effect of forest fragmentation on the soil seed bank of central amazonia)," vol. 393, no. Supplement C, pp. 105-112, 2017.

[43] O. Vargas and F. Mora, Estrategias para la restauración ecológica del Bosque altoandino: el caso de la Reserva Forestal Municipal de Cogua, Cundinamarca. Universidad Nacional de Colombia, Facultad de Ciencias, Departamento de Biología, 2007.

[44] R. I. V. Caicedo, K. J. D. Guarín, and Y. R. Perdomo, "Composición y diversidad del banco de semillas en áreas urbanas fragmentadas de piedemonte, villavicencio, colombia," Ingenierías USBMed, vol. 9, no. 1, pp. 86-96, 2018.

[45] R. Velosa, J. Gonzalez, J. Pardo, and Acosta, Viabilidad ecológica y técnica para el establecimiento de un área de jardín botánico en predios de la Universidad Santo Tomás, sede Loma Linda, Villavicencio. Villavicencio: Universidad Santo Tomás, 2017.

[46] R. D. R. Acevedo, "Capacidad de carga turística del área de uso público del parque ecológico el samán. cartago, valle." Tesis de pregrado, Universidad Tecnológica de Pereira, 2012.

[47] M. Cifuentes, Determinación de capacidad de carga turística enáreas protegidas. No. 194, Bib. Orton IICA/CATIE, 1992.

[48] P. D. Bianchi, "Acerca de la refuncionalización de objetos," Actas de Diseño. Facultad de Diseño y Comunicación. Universidad de Palermo. ISSN, vol. 8, pp. 10-12, 2014.

[49] G. Mendiola, "Estudio axiológico estético de la arquitectura en méxico a finales del siglo xx (19601990)." Tesis doctoral, Universidad Autónoma del Estado de Morelos, México, 2007.

[50] AIP Asociación para la interpretación del patrimonio, "Boletín de interpretación," no. 6, 2002.

[51] M. G. Linkies, "La importancia de las personas en la preservación de la naturaleza," Ingenierías
USBMed, vol. 2, no. 1, pp. 5-7, 2011.

[52] Alcaldía de Villavicencio, "Síntesis diagnóstica: Plan de ordenamiento territorial pot." Disponible en: http://antigua.villavicencio.gov.co, 2015. 